\title{
ARTICLE OPEN \\ Smart machine learning or discovering meaningful physical and chemical contributions through dimensional stacking
}

\author{
Lee A. Griffin (iD ${ }^{1,2}$, laroslav Gaponenko ${ }^{2,3}$, Shujun Zhang iD $^{4}$ and Nazanin Bassiri-Gharb $\mathbb{D}^{2,5}$
}

\begin{abstract}
Despite remarkable advances in characterization techniques of functional materials yielding an ever growing amount of data, the interplay between the physical and chemical phenomena underpinning materials' functionalities is still often poorly understood. Dimensional reduction techniques have been used to tackle the challenge of understanding materials' behavior, leveraging the very large amount of data available. Here, we present a method for applying physical and chemical constraints to dimensional reduction analysis, through dimensional stacking. Compared to traditional, uncorrelated techniques, this approach enables a direct and simultaneous assessment of behaviors across all measurement parameters, through stacking of data along specific dimensions as required by physical or chemical correlations. The proposed method is applied to the nanoscale electromechanical relaxation response in $(1-x)$ PMN-xPT solid solutions, enabling a direct comparison of electric field- and chemical composition-dependent contributors. A poling-like, and a relaxation-like behavior with a domain glass state are identified, and their evolution is tracked across the phase diagram. The proposed dimensional stacking technique, guided by the knowledge of the underlying physics of correlated systems, is valid for the analysis of any multidimensional dataset, opening a spectrum of possibilities for multidisciplinary use.
\end{abstract}

npj Computational Materials (2019)5:85 ; https://doi.org/10.1038/s41524-019-0222-z

\section{INTRODUCTION}

Functional materials have been at the core of exponential advances in technology over the last century, in wearable electronics, ${ }^{1-3}$ batteries, ${ }^{4,5}$ optoelectronic systems, ${ }^{6,7}$ and various microelectronic devices, 8,9 among others. However, the development of new functional materials has often hinged on trial-anderror based chemical doping of preexisting chemistries, as opposed to deliberate and informed design. Such an approach has often stemmed from an incomplete understanding of the physical and chemical interplay resulting in the functional response at the micron and submicron length scales.

An excellent example is offered by relaxor-ferroelectric single crystals that exhibit exceptionally large piezoelectric coefficients at the morphotropic phase boundary (MPB), when cut and poled along specific crystallographic directions. ${ }^{10}$ One such material, $(1-x) \mathrm{Pb}\left(\mathrm{Mg}_{1 / 3} \mathrm{Nb}_{2 / 3}\right) \mathrm{O}_{3}-x \mathrm{PbTiO}_{3}$ or $(1-x) \mathrm{PMN}-x \mathrm{PT}$, has been of particular interest for acoustic transduction applications, such as underwater sonars and medical ultrasound devices. ${ }^{11,12}$ Starting from a pure relaxor at $x=0$, and by creation of a solid solution with the ferroelectric end-member $\left(\mathrm{PbTiO}_{3}\right)$, the material exhibits an evolution of the electromechanical properties, through an MPB at $x \approx 31-35 \% .^{13}$ While still under debate, the origin of the remarkable piezoelectric response has been ascribed to several contributors, including the presence of the relaxor end-member, large shear piezoelectric response, ${ }^{14}$ structural heterogeneities ${ }^{15}$ that can contribute to the electrical and mechanical field induced phase transitions, ${ }^{16}$ and ergodic and nonergodic polar and chemical nanoregions (PNR and CNR). ${ }^{17,18}$ Hence, since their discovery two decades ago, this limited understanding of the nanoscale chemophysical interactions resulting in the enhanced functionalities has also limited further development of new compositions to chemical doping. ${ }^{19-22}$

With the advent of characterization techniques that allow in situ and operando observations of the materials at these length scales, it has finally become possible to tackle some of the most challenging questions about the origin of the functional response in materials with complex chemistry. For instance, advances in high-resolution transmission electron microscopy (TEM) have enabled investigation of the structure and properties of relaxorferroelectrics and lead-free ceramics under the influence of mechanical stress, ${ }^{23,24}$ electric field, ${ }^{25,26}$ or temperature. ${ }^{27}$ One such study ${ }^{25}$ identified a co-dependence between applied electrical and mechanical fields, ferroelastic nanodomains, and the coercive fields of ferroelectric domains in PMN-0.36PT single crystals. Others have reported application of in situ TEM characterizations for investigation of reversible and irreversible ferroelectric domain switching induced by mechanical stress. ${ }^{23,24}$ In addition, piezoresponse force microscopy (PFM) has been used to study the local electromechanical response at the nanoscale in relaxor-ferroelectrics ${ }^{28-32}$-exploring both the role of the applied electric field on the switching behavior and polarization dynamics, ${ }^{33,34}$ as well as the temperature dependence of domain structures $^{35}$ and mesoscale phase transitions. ${ }^{36}$

Data generated through these methods is often of large complexity, and thus allows limited insight from direct inspection and traditional statistical analysis. The recent developments for

\footnotetext{
${ }^{1}$ School of Electrical Engineering, Georgia Institute of Technology, Atlanta, GA, USA; ${ }^{2}$ G.W. Woodruff School of Mechanical Engineering, Georgia Institute of Technology, Atlanta, GA, USA; ${ }^{3}$ Department of Quantum Matter Physics, University of Geneva, Geneva, Switzerland; ${ }^{4}$ ISEM, Australian Institute for Innovative Materials, University of Wollongong, Wollongong, Australia and ${ }^{5}$ School of Materials Science and Engineering, Georgia Institute of Technology, Atlanta, GA, USA

Correspondence: Nazanin Bassiri-Gharb (nazanin.bassirigharb@me.gatech.edu)

These authors contributed equally: Lee A. Griffin, laroslav Gaponenko
}

Received: 11 February 2019 Accepted: 22 July 2019

Published online: 12 August 2019 
application of big data analytics techniques to materials science have offered a path forward, providing a powerful new approach to handle and analyze the information. Specifically, dimensional reduction $(D R)$ and machine-learning $(\mathrm{ML})$ techniques have been hailed as a groundbreaking paradigm in materials science, ${ }^{37}$ and used to identify superimposed physical and chemical contributors to functional behavior within multidimensional datasets in fields as diverse as multiferroics, ${ }^{38,39}$ superconductors, ${ }^{40}$ oxide interfaces, ${ }^{41}$ and electromechanically active materials. ${ }^{42-45}$ However, while these techniques hold significant promise to revolutionize our understanding of the fundamental material science and guide future design of materials, they are inherently limited by a lack of means to impose physical or chemical constraints to the analysis. $^{46}$ In particular, conventional DR analysis on high dimensional datasets is often performed through creation of two-dimensional data slices, where only one single parameter is changed within the slice.

In this work, an approach to encode meaningful chemical and physical boundary conditions in ML and DR analysis of multidimensional datasets is formally presented. While the conventional DR approach employs independent analysis of data slices, here we stack, or concatenate, the slices along appropriate dimensions (axes), before DR is applied. Physical and chemical constraints are thus implicitly imposed as correlation across parameters used for stacking of data slices. Compared to other techniques, such as averaging, the proposed approach offers a quantitative and lossless comparison of behaviors (eigenvectors) across measurement parameters. Its versatility and ease-of-use are demonstrated through the analysis of the electromechanical response in $(1-x)$ PMN-xPT solid solutions as a function of electric field and composition, revealing the evolution and interplay of the mechanisms underpinning the observed local functionality.

\section{RESULTS AND DISCUSSION}

\section{Relaxation}

The local electromechanical response of 001-cut pure relaxor PMN, near-MPB PMN-0.36PT and relaxor ferroelectric PMN-0.40PT single crystals was probed by band excitation PFM (BE-PFM) in order to investigate the evolution of the piezoresponse amplitude as a function of time, voltage, and composition across the phase diagram. A $50 \times 50$ grid (2500 different locations) were probed by BE-PFM for each composition over a $2 \mu \mathrm{m} \times 2 \mu \mathrm{m}$ sample area. Each measurement was made with application of a pulse train of alternating polarity and increasing voltage pulses, spanning below and above the coercive voltage. The time-dependent relaxation of the electromechanical response was measured at the trailing edge of each voltage pulse. This measurement approach is illustrated in Fig. S1 in the Supplementary Information.

The complete experiment resulted in a five-dimensional dataset $D\left(t, x, y, p_{v}, p_{c}\right)$ of over a million individual data points, as a function of time $t$, the two spatial dimensions $x, y$ describing the sampled areas, applied voltage $p_{v}$, and composition $p_{c}$. Henceforth, the two spatial dimensions are reduced to a single location identifier (i.e., $z=[x, y]$ ) for ease of handling. In the past, such datasets have been analyzed with conventional DR by slicing the data into voltage datasets for each composition, $D^{p_{v}, p_{c}}(t, z)$, and analyzing the two-dimensional slices independently. ${ }^{42-45}$ Conversely, applying DR with dimensional stacking concatenates the $D^{p_{v}, p_{c}}(t, z)$ slices along appropriate axes prior to DR analysis, based on physical and chemical understanding of the material. Here, we compare the resulting phenomenological insights obtained through traditional statistical analysis, the conventional DR approach, and DR with dimensional stacking.
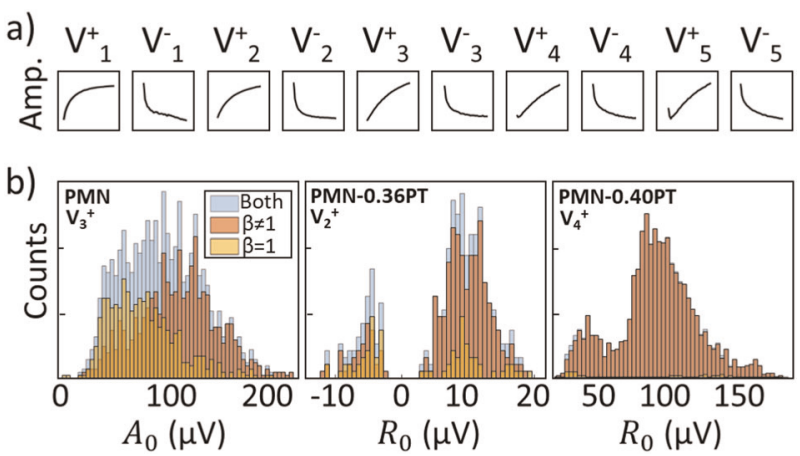

Fig. 1 Simple statistical analysis of the data, through simple averaging and histogram distribution. a Average time-dependent piezoresponse amplitude of the pure relaxor PMN as a function of voltage, averaged over all 2500 points. b Distributions of: PMN's $A_{0}$ for the response to $V_{3}^{+}, \mathrm{PMN}-0.36 \mathrm{PT}$ 's $R_{0}$ for the response to $V_{2}^{+}$, and PMN-0.40PT's $R_{0}$ for the response to $V_{4}^{+}$. $A_{0}$ is the piezoresponse amplitude at saturation, $R_{0}$ is the overall amplitude change over time, and $\beta$ is the stretch exponential

\section{Statistical analysis}

The simplest approach to a quantitative comparison between the various compositions $\left(p_{c}\right)$ and applied voltages $\left(p_{v}\right)$ is to average the response $D^{p_{v}, p_{c}}(t, z)$ over the spatial dimensions, resulting in a time-dependent piezoresponse $D^{p_{v}, p_{c}, \bar{z}}(t)$ for any given voltage and composition. The complete results for all three compositions are shown in Fig. S2 in the Supplementary Information. The average piezoresponse evolution of pure relaxor PMN as a function of time after voltage removal is shown in Fig. 1a for each voltage. Two fundamentally different behaviors are observed, correlated with the voltage polarity: for positive voltages, the piezoresponse increases over time; for negative voltages, it decays over time. Based on their similarity with existing processes in ferroelectric materials, the decay and increase will henceforth be referred to as relaxation and poling, respectively.

A comparison of the averaged response across all voltages and compositions (Fig. S3 in the Supplementary Information), shows that the poling behavior is observed almost exclusively in the pure relaxor end member. Such relaxation and poling behaviors in relaxor-ferroelectric systems have been previously analyzed through a stretched exponential decay, or Kohlrausch-William-Watts (KWW) function,

$\operatorname{KWW}(t)=A_{0}+R_{0} e^{\left(-(t / \tau)^{\beta}\right)}$,

where $A_{0}$ is the response at saturation, $R_{0}$ is the amplitude of the change over time, $\tau$ is the characteristic time constant, and $\beta$ is the stretch exponential, describing the width of the time constant distribution. ${ }^{43}$ When $\beta \rightarrow 0$, the time constant distribution is the widest, and when $\beta \rightarrow 1$, the $\tau$ distribution is the tightest, with a simple exponential decay at the limit of $\beta=1$. The KWW functional has been successfully applied to many out-ofequilibrium systems, with an anticorrelation between the disorder and $\beta{ }^{47,48}$ The fitting coefficients for a KWW analysis of the averaged piezoresponse over time for each composition and voltage are reported in Figs S4-S9 in the Supplementary Information (Figs S4 and S5 plot PMN's response to positive and negative applied voltages, respectively, Figs S6 and S7 plot PMN$0.36 \mathrm{PT}$ 's response to positive and negative applied voltages, respectively; and Figs S8 and S9 plot PMN-0.40PT's response to positive and negative applied voltages, respectively). However, while such an approach benefits from the reduced statistical noise due to spatial averaging, it results in a loss of any spatial information and therefore possible local chemical heterogeneities.

In order to retain the spatial information, KWW fitting can be performed individually for all of the probed points for each 
a)
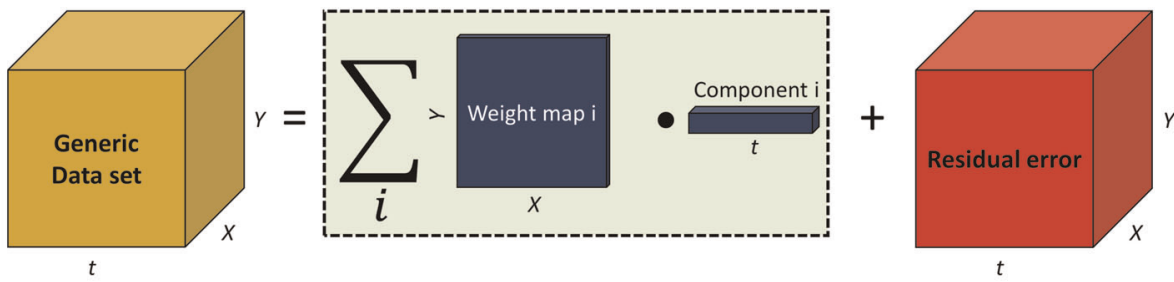

b)

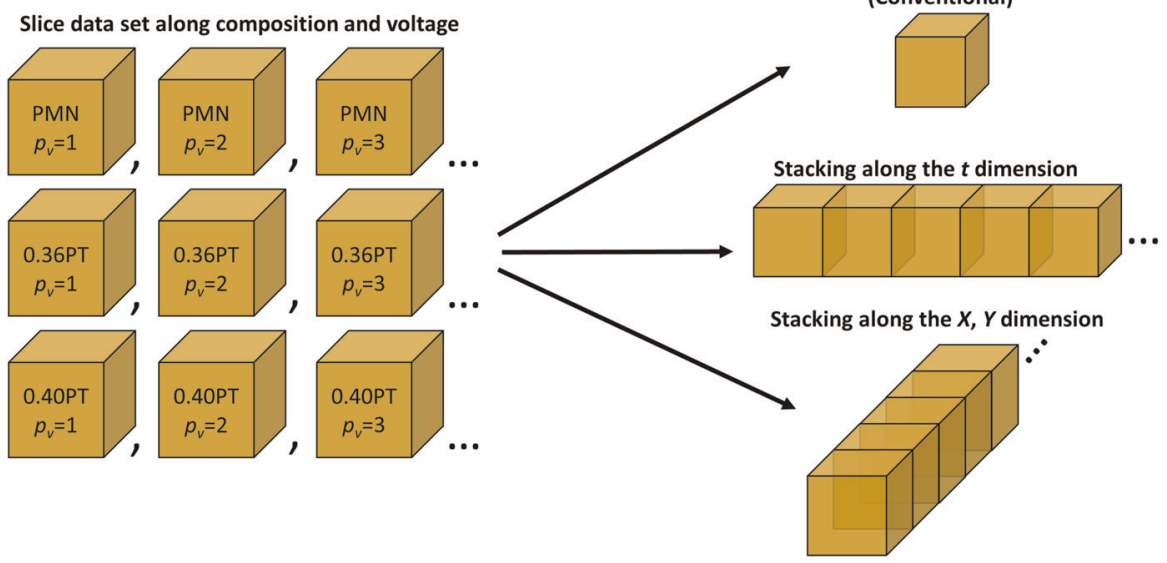

c)

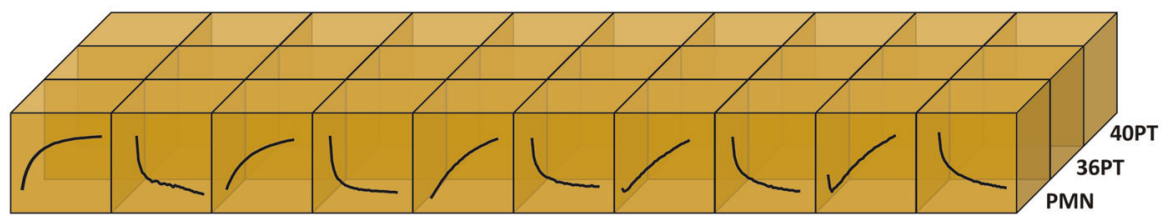

Fig. 2 Illustrations of $\mathbf{a}$ the desired outcome of dimensional reduction for a generic dataset dependent on space and time, $\mathbf{b}$ the various approaches to dimensional reduction on the $D\left(t, z, p_{v}, p_{c}\right)$ dataset, and $\mathbf{c}$ the voltage- and composition-stacked data. In general, the goal in this work is to identify time-dependent fundamental behaviors and spatial weightings of those behaviors. This is complicated by the inclusion of additional measurement parameters, $p_{v}$ and $p_{c} . \mathbf{b}$ The dataset can be sliced into subsets and analyzed independently (top arrow), as is done conventionally. Alternatively, dimensional stacking can be performed along the spatial or temporal axis (middle and bottom arrows, respectively). c Dimensional stacking can be extended to both the $p_{v}$ and $p_{c}$ parameters. Here, the composition parameter $\left(p_{c}\right)$ is $s p a t i a l l y$ stacked and the voltage parameter $\left(p_{v}\right)$ is temporally stacked

sample, albeit with greater error margins. The distribution of select KWW coefficients, samples, and at specific applied voltages are shown in Fig. 1b. The observed bimodal distributions-e.g., in the $R_{0}$ coefficients for the $V_{4}^{+}$voltage of PMN-0.40PT-highlight the presence of more than one relaxation behavior for a single voltage. The coexistence of differing contributors to the response is obviously lacking in the information provided by averaging. Furthermore, while both the averaged response and the statistical distributions identify the presence of different fundamental behaviors across compositions and voltages, they also fail to identify direct quantitative commonalities and/or any spatial correlation. This fundamental limitation can be resolved through use of data analytics techniques and specifically DR approaches.

\section{Dimensional reduction}

Dimensional reduction applies nonlinear statistical and algebraic methods to the input dataset $D$ in order to extract a basis that describes it in a user-specified number, $N$, of eigenvectors (also called components), $\varepsilon_{i}$, and their associated weights (also called loading maps or mixing matrices), $w_{i}$, yielding:

$D=\sum_{i=0}^{N} w_{i} \varepsilon_{i}+$ error

with a residual error that will depend on the dataset quality, method used, convergence criterion, and $N$. In the ideal case, once this decomposition has been successfully performed, $\varepsilon_{i}$ will describe specific fundamental behaviors (physical and/or chemical), and their relative abundance at different spatial points over the sample areas will correspond to $w_{i}$.

As discussed, it is possible to apply the DR methods to the fourdimensional relaxation dataset $D\left(t, z, p_{v}, p_{c}\right)$. As the probed physical quantities are time-dependent, the resulting behaviors (or components) will have to be expressed as a function of time, $\varepsilon_{i}(t)$. Similarly, the weights should depend on space, $w_{i}(z)$, enabling their representation as spatial weight maps for simple visual inspection of the relative contribution of the resulting behaviors to the response over the probed area. This is illustrated in Fig. 2a. Therefore, the data are divided into two-dimensional slices of individual voltage and composition combinations to be analyzed independently, i.e.,

$$
D^{p_{v}, p_{c}}(t, z)=\sum_{i=0}^{N} w_{i}^{p_{v}, p_{c}}(z) \varepsilon_{i}^{p_{v}, p_{c}}(t)+\text { error. }
$$

Equation (3) expresses in its simplest form a conventional DR approach for the acquired relaxation dataset, resulting in a total of $N \times 10 p_{v} \times 3 p_{c}=30 \times N$ components with a corresponding weight map for each component. The conventional approach is illustrated by the top arrow in Fig. 2 b. 

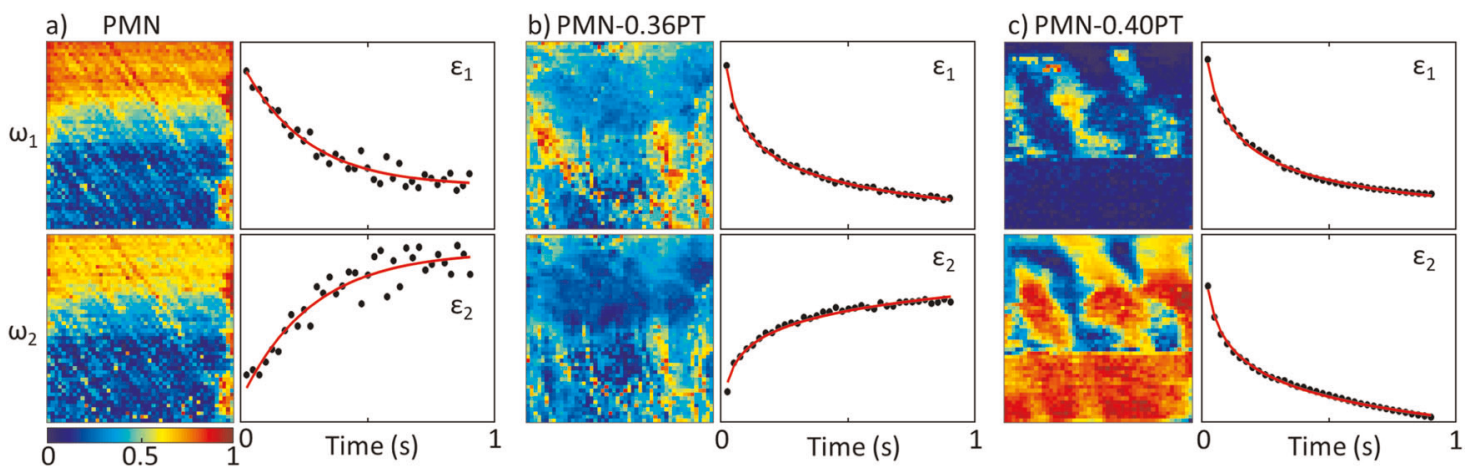

Fig. 3 Two-component conventional NMF analysis. Eigenvectors and weight maps are shown for conventional dimensional reduction, NMF for a PMN, b PMN-0.36PT, c PMN-0.40PT at an applied voltage of $\approx 2-3 V_{c}^{-}$. The weight maps $\left(\omega_{1}\right.$ and $\left.\omega_{2}\right)$ represent the abundance of the corresponding eigenvectors $\left(\varepsilon_{1}\right.$ and $\left.\varepsilon_{2}\right)$ over the probed sample areas, i.e., the $x$ - and $y$-directions in the weight maps represent the $x$ - and $y$ directions in the sampled area. The red lines represent the KWW fit to each curve

For the analysis, the number of components $N=2$, was selected to account for the bimodal behavior evidenced in the previous statistical analysis. Nonnegative matrix factorization (NMF) was chosen as the specific method of DR. NMF is well suited to this work, as the component/weight pairs that it produces are strictly nonnegative-reflecting the inherent additive behavior of the data, and thus enabling a clear separation of relaxation and poling behaviors.

Figure 3 depicts the resulting first and second components and their respective weight maps for all samples at a single selected applied voltage $p_{v} \approx 2 V_{c}^{-} 3 V_{c}^{-}$(where $V_{c}^{-}$is the negative coercive voltage identified through initial piezoresponse force switching spectroscopy measurements for each sample), and the red lines represent the KWW fit to each curve. It is worth noting that NMF identifies a co-presence of poling and relaxation behavior in the PMN sample, whereas the average response in Fig. 1a showed only a relaxation for PMN in response to negative voltages $\left(V_{i}^{-}\right)$. Such a behavior can either come from a discrete physical process or from over-parametrization due to the number of components $N$ being too large. Subsequent KWW analysis was performed on each extracted component $\varepsilon_{i}$ to quantitatively compare the response contributors across the compositions. The fitting parameters are reported in Tables S1 and S2 in the Supplementary Information. The specific value of the time-dependent piezoresponse of the relaxor end member PMN in Fig. 3a corresponds to a simple exponential decay $(\beta=1)$ for both components. This behavior substantially changes with the addition of the ferroelectric $\mathrm{PbTiO}_{3}$ end-member to the solid solution. For both PMN0.36PT and PMN-0.40PT, illustrated in Fig. 3b, c, respectively, the $\beta$ exponents range from 0.50 to 0.63 , suggesting wide decay time distributions. It is noteworthy that such stretch exponents are characteristic of domain glasses. ${ }^{49,50}$

Additional inspection shows that both PMN in Fig. 3a and PMN$0.36 \mathrm{PT}$ in Fig. $3 \mathrm{~b}$ exhibit poling behavior in the NMF analysis. However, each of the $30 \times N$ component-weight pairs (3 compositions, 10 voltages) is independently generated, disregarding any $p_{v}$ (voltage), or $p_{c}$ (composition) correlation. Therefore, even simple comparison of results across different voltages and/or compositions is made impossible by lack of common behaviors: due to the pair-wise nature of the DR (weight map and its corresponding component), a direct comparison between two or more $\varepsilon_{i}$ is hindered by "scaling" through the corresponding $\omega_{i}$. While indirect comparison by proxy is possible through fitting of individual components to a physical model, this intrinsic limitation hinders more advanced analysis of correlations. In brief, conventional DR provides a behavioral-spatial correlation lacking in traditional statistical analysis, but a method for analyzing the data as a whole is necessary for correlation across all parameters.

\section{Dimensional stacking}

Quantitative comparative analysis of the entire dataset can be achieved through dimensional stacking prior to DR. Dimensional stacking is performed by concatenating the $D^{p_{v}, p_{c}}(t, z)$ slices prior to analysis, as shown previously. ${ }^{43}$ Such data-slice concatenations can be realized if the material is known to exhibit correlative behavior across the physical or chemical parameters involved. Here, we formally define dimensional stacking and demonstrate how the constraints are imposed and affect the subsequent interpretation of the resulting eigenvectors and weight maps.

To demonstrate the proposed method, we consider PMN0.36PT: the choice of a single composition translates into selection of the data slice with $p_{c}=2$, i.e., $D^{p_{v}, p_{c}=2}(t, z)$. The $p_{v}$ slices in this dataset can be concatenated into a single dataset by stacking them along either the temporal or the spatial dimension, as illustrated by the middle and bottom arrows of Fig. 2b, respectively. This approach inherently imposes constraints to the DR analysis: concatenation of datasets along the temporal dimension will impose a spatial constraint, and concatenation along the spatial dimension will impose a temporal correlation. For instance, let's consider concatenation of $p_{v}$ slices along the time dimension (middle arrow of Fig. 2b):

$$
D^{p_{c}=2}(t, z)=\left[\begin{array}{c}
D^{p_{v}=1, p_{c}=2}(t, z) \\
D^{p_{v}=2, p_{c}=2}(t, z) \\
\cdots \\
D^{p_{v}=10, p_{c}=2}(t, z)
\end{array}\right] .
$$

Dimensional reduction analysis of this dataset results in the eigenvectors inheriting a $p_{v}$ dependence. Thus, the material's response is collectively derived as a series of concatenated $p_{v}$ behaviors:

$$
D^{p_{c}=2}(t, z)=\sum_{i=0}^{N} w_{i}^{p_{c}=2}(z) \varepsilon_{i}^{p_{c}=2}\left(t, p_{v}\right)+\text { error. }
$$

Such a concatenation implies that at every location the material responds in a correlated way to the train of voltages ( $p_{v}=1$ to 10 ) seen over time (i.e., the electromechanical response of the material is correlated at sub- and super-coercive probing voltages). This physical correlation would seem not only possible, but indeed logical, as fundamental contributors to the observed piezoresponse (e.g., nonlinear, ferroelectric behavior, and charge injection) are all expected to show specific electric fielddependent behaviors.

Figure 4 a shows the results of the $p_{v}$ dimensional stacking along the time dimension for the two-component NMF analysis of PMN0.36PT: the time-dependent components, $\varepsilon_{i}$, now encode the voltage dependence alongside the time evolution thereof. 
a)

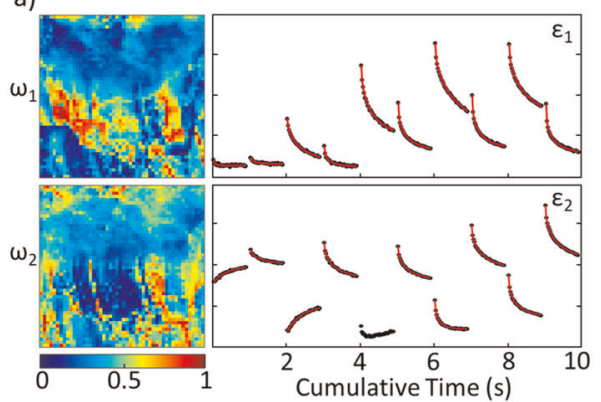

b)

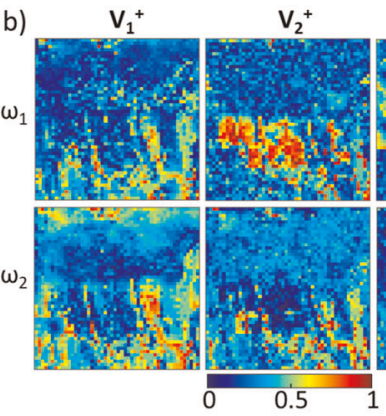

$\mathrm{V}^{+}$

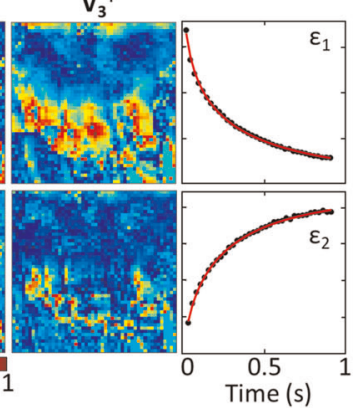

Fig. 4 Components $\left(\varepsilon_{1}\right.$ and $\left.\varepsilon_{2}\right)$ and weight maps $\left(\omega_{1}\right.$ and $\left.\omega_{2}\right)$ obtained from a two-component NMF analysis of PMN-0.36PT with voltage stacking along the $\mathbf{a}$ time or $\mathbf{b}$ spatial dimension, with weight maps representing the abundance of the corresponding components over the probed area. The red lines represent KWW fits. Note that in $\mathbf{b}$ only the weight maps for the first three positive pulses are shown. The voltage stacking results in a spatial or $\mathbf{b}$ temporal correlation at the different voltages. a Voltage stacking along the time dimension implies that the material in a single location responds in a correlated way to the train of voltages seen over time (i.e., correlated sub- and super-coercive response). Here, the eigenvector components inherit the $p_{v}$ dependence, thus, the material's response to all $p_{v}$ is collectively derived as a series of concatenated behaviors. b Voltage stacking along the space dimension implies a single underlying physical response (eigenvector) across all the voltages, which can develop differently across the sampled area at each applied voltage. Here, the weight maps inherit the $p_{v}$ dependence, i.e., the eigenvectors are voltage-independent, but a weight map is generated for each $p_{v}$ value

Component $1, \varepsilon_{1}$ shows only relaxation across all voltages with an increasing baseline and amplitude. However, the second component $\varepsilon_{2}$ shows relaxation at negative applied voltages and higher positive voltages only, with poling being present at lower positive voltages. In addition, while the first component's relaxation behavior tends to saturate as voltage magnitude increases (at both positive and negative polarity), the second component's response is far from saturation and grows continuously over the voltage range studied. Lastly, we note that the weight maps differ from those shown in Fig. 3 for the same composition, as the new results encompass spatial correlation of the behaviors across all voltages.

Alternatively, we can consider a concatenation of $p_{v}$ slices along the spatial dimension (bottom arrow of Fig. 2b):

$$
D^{p_{c}=2}(t, z)=\left[\begin{array}{llll}
D^{p_{v}=1, p_{c}=2}(t, z) & D^{p_{v}=2, p_{c}=2}(t, z) & \ldots & D^{p_{v}=10, p_{c}=2}(t, z)
\end{array}\right] .
$$

Dimensional reduction analysis of this dataset results in the weight maps inheriting a $p_{v}$ dependence, with common eigenvectors for all $p_{v}$ values:

$D^{p_{c}=2}(t, z)=\sum_{i=0}^{N} w_{i}^{p_{c}=2}\left(z, p_{v}\right) \varepsilon_{i}^{p_{c}=2}(t)+$ error.

Hence, the DR analysis is constrained to consider that the response of the material to all voltages is temporally correlated. This implies that a single physical behavior (eigenvector) underlies all the voltages, and can develop differently across the sampled area at each applied voltage. Here, the weight maps inherit the $p_{v}$ dependence, i.e., the eigenvectors are voltage-independent, but a weight map is generated for each $p_{v}$ value.

Figure $4 \mathrm{~b}$ shows the results of the $p_{v}$ dimensional stacking along the spatial dimension for the two-component NMF analysis of PMN-0.36PT. Similar to the conventional DR approach above (Fig. 3), component 1 and component $2\left(\varepsilon_{1}\right.$ and $\left.\varepsilon_{2}\right)$ show relaxation and poling, respectively. The weight maps (shown for the first three positive voltages) depict an expansion of the areas with highcontributing component 1 (relaxation) at increasing voltage magnitude, accompanied by a contraction of the areas where component 2 (poling) is contributing. Note that the $\varepsilon_{1}$ and $\varepsilon_{2}$ in Fig. 4 are presented with $y$-axis values in Figs $S 10$ and $S 11$ in the Supplementary Information.

In order to further identify behaviors associated with the presence of relaxor PMN and ferroelectric $\mathrm{PbTiO}_{3}$ end members, it is also possible to apply dimensional stacking to the composition parameter. Specifically, in our case, composition-dimensional stacking was performed along the spatial dimension in order to obtain weight maps with an inherited chemical compositiondependence. Such a dimensional stacking imposes a behavioral constraint across the phase diagram in the solid solution. In addition, combining chemical composition stacking with voltage stacking along the time dimension allows us to explore the evolution of the physical processes not only as a function of voltage but also across different compositions. The combined chemical and physical dimensional stacking dataset results in a single matrix (illustrated in Fig. 2c), with the tightest chemical and physical constraints for analysis:

$$
\mathbf{D}(t, z)=\left[\begin{array}{ccc}
D^{p_{c}=1, p_{v}=1}(t, z) & D^{p_{c}=2, p_{v}=1}(t, z) & D^{p_{c}=3, p_{v}=1}(t, z) \\
D^{p_{c}=1, p_{v}=2}(t, z) & D^{p_{c}=2, p_{v}=2}(t, z) & D^{p_{c}=3, p_{v}=2}(t, z) \\
\ldots & \ldots & \ldots \\
\ldots & \cdots & \ldots \\
D^{p_{c}=1, p_{v}=10}(t, z) & D^{p_{c}=2, p_{v}=10}(t, z) & D^{p_{c}=3, p_{v}=10}(t, z)
\end{array}\right],
$$

which with DR provides:

$$
D(t, z)=\sum_{i=0}^{N} w_{i}\left(z, p_{c}\right) \varepsilon_{i}\left(t, p_{v}\right)+\text { error. }
$$

Figure 5 shows the results of a two-component NMF analysis of the combined voltage- and composition-stacked dataset. Component $1, \varepsilon_{1}$, shows strong relaxation behavior at all voltages, somewhat tending to saturate at increasing applied voltage amplitudes. This component is notably strong in the PMN-0.36PT and PMN-0.40PT compositions and significantly absent in the pure relaxor. Conversely, the second component $\left(\varepsilon_{2}\right)$, with strong poling at $V_{i}^{+}$and weak relaxation at $V_{i}^{-}$, is observed throughout the phase diagram, at varying intensities. Furthermore, this component is reminiscent of the averaged response of PMN shown in Fig. 1a, in that it shows poling in response to positive voltages. These components are shown with $y$-axis values in Fig. S12 in the Supplementary Information.

Quantitative information can also be extracted from the components by fitting the individual behaviors to the KWW function. The resulting coefficients are plotted in Fig. 6. For ease of fitting, the responses to the highest positive voltages of the second component were considered as pure poling, ignoring the first three experimental data points for each curve. The error bars (representing confidence intervals) are subsequently larger for these data points, indicating a possible contribution from yet a third mechanism. The second component's response to the first negative voltage was not fitted as it showed no time dependence. 


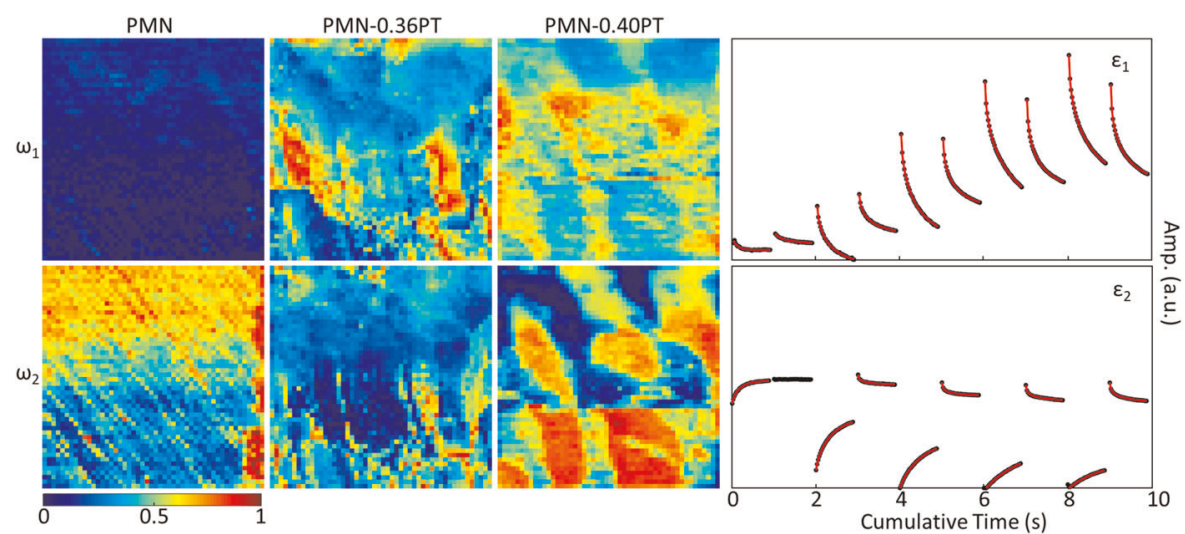

Fig. 5 Voltage- and composition-stacking in NMF analysis. Eigenvectors $\left(\varepsilon_{1}\right.$ and $\left.\varepsilon_{2}\right)$ and weight maps $\left(\omega_{1}\right.$ and $\left.\omega_{2}\right)$ are shown for a twocomponent NMF on the voltage- and composition-stacked dataset for PMN, PMN-0.36PT, and PMN-0.40PT. The $D^{p_{c}, p_{v}}(t, z)$ slices are voltagestacked along the time axis, resulting in the components inheriting the $p_{v}$ dependence (similar to Fig. 4a). These slices are subsequently composition-stacked along the spatial axis, resulting in the weight maps inheriting the $p_{c}$ dependence (similar to Fig. $4 \mathrm{~b}$ ). The red lines represent the KWW fitting to each curve

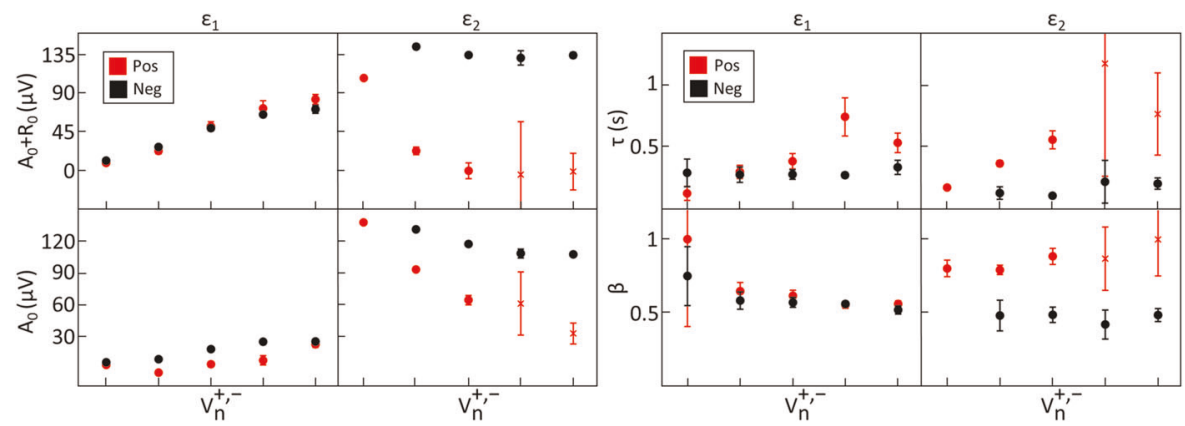

Fig. 6 KWW functional fitting parameters for the voltage- and composition-stacked NMF eigenvectors. Functional parameters were extracted from the combined dimensional stacking analysis (Fig. 5) for both component $1\left(\varepsilon_{1}\right)$ and component $2\left(\varepsilon_{2}\right)$ and at each applied voltage. $A_{0}+R_{0}$ is the total piezoresponse amplitude, $A_{0}$ is the piezoresponse amplitude at saturation, $\tau$ is the characteristic time constant, and $\beta$ is the stretch exponential. The bars represent the confidence intervals

The response at saturation $\left(A_{0}\right)$ and initial piezoresponse value $\left(A_{0}+R_{0}\right)$ plots confirm that the response to negative voltages in component 1 tends to saturate with increasing applied voltage amplitude. The stretch exponential $\beta$ quickly approaches $\approx 0.6$ in component 1 for either voltage polarity, and is approximately equal to $0.5-0.6$ in response to negative voltages for the second component, showing a relaxation behavior. Conversely, $\beta$ steadily increases with voltage amplitude from 0.75 to 1 in response to positive voltages for the second component, exhibiting a poling behavior.

A comparison of the different methods used to analyze the multivariate data highlights the advantages and disadvantages associated with each. Averaging and statistical distribution analysis successfully identified the presence of at least two distinct contributors, but could not directly identify these behaviors. Application of conventional DR allowed for identification of behaviors and the respective spatial mapping thereof. However, this approach could not easily provide quantitative comparisons across all the parameters, precluding correlative analysis. $P_{V}$ dimensional stacking for a single composition enabled such a comparison. Stacking along the time dimension (Fig. 4a) generated unique voltage-correlated behaviors, while stacking along the spatial dimension tracked the evolution of shared behaviors with increasing voltage magnitudes (Fig. 4b). Lastly, the combined voltage- and composition-stacked DR produced a quantitative correlation of piezoresponse evolution across the sampled areas, as a function of applied voltages and probed chemical compositions.
Furthermore, regardless of the analysis method, a poling-like behavior was observed, most strongly correlated with the relaxor end member, PMN. Referring back to Fig. 3, the weight maps for the two components (relaxation and poling) are very similar, while the actual components tend to reproduce almost symmetric local trends. Consistently with the voltage- and composition-stacked analysis shown in Fig. 5, it is clear that only a single behavior is present in the pure PMN sample: relaxation in response to negative applied voltages and poling-like response to positive applied voltages. Hence, this behavior can be considered an indisputable signature of the relaxor end member, and indeed persists even at ferroelectric addition to the solid solution. We note that these results are consistent with previous work finding that the relaxor-type behavior persists into compositions with substantial ferroelectric content, i.e., as high as PMN-0.5PT. ${ }^{51}$

In addition, the dimensional stacking analysis clearly shows that two factors correlate with the appearance of a relaxationdominated behavior: namely, introduction of the ferroelectric end member and applied field magnitude. As demonstrated in component 1 of Fig. 5, a strong relaxation in the piezoresponse, reminiscent of typical ferroelectric relaxation, is observed with introduction of the ferroelectric end member. Such relaxation saturates with increasing magnitude of the applied voltage, at values well above the $V_{c}^{ \pm}$. The weight maps in Fig. $4 \mathrm{~b}$ depict an enhancement in the relaxation behavior and a decrease in the poling behavior when the voltage magnitude is increased.

Finally, it should be noted that relaxations with $\beta$ values approximately equal to 0.6 have been associated with domain 
glasses, ${ }^{49,50}$ or domains with glassy properties. In general, glasslike behavior is associated with complex nonequilibrium states of matter with strong disorder. ${ }^{52}$ However, the disorder is often associated with lack of structural organization. In comparison, here glassy behavior is observed in bulk single crystals, where the disorder is observed for the functional (i.e., physical) response of the material, as a direct result of local chemical and polar heterogeneities. In fact, the glassy domain behavior is persistently observed when a ferroelectric solid solution is created with the relaxor end member. Although glassy behavior, i.e., dipolar- and spin-glass behavior, has been reported extensively in literature for relaxor materials, such reports have been limited to either pure relaxor (e.g., PMN) or relaxor-ferroelectric solid solutions with small ferroelectric content. ${ }^{53}$ Here, the dimensional stacking analytic techniques highlight specifically that the domain glass behavior can be observed throughout the phase diagram, again consistent with literature. ${ }^{51}$ Further, this domain glass behavior is a strong signature of the relaxor-ferroelectric solid solutions, as well as perceivably of the very large enhancement in electromechanical response observed in these materials.

In summary, a method for applying physical and chemical constraints to $\mathrm{ML}$ techniques is presented. Through informed use of dimensional stacking, meaningful chemical and physical characteristics from multidimensional datasets can be extracted. Thanks to its intrinsically correlative nature, this method outperforms conventional DR and statistical techniques that are limited by information loss or lack direct comparability.

To demonstrate the dimensional stacking approach, nanoscale investigations of electromechanical relaxation of $(1-x) \mathrm{PMN}-x \mathrm{PT}$ relaxor-ferroelectrics across the phase diagram $(x=0,0.36,0.4)$ were performed. Specifically, simple statistical analysis, conventional DR, and finally dimensional stacking techniques were leveraged to identify the multiple contributors to the observed electromechanical response at different ferroelectric end member contents. All approaches identified both poling and relaxation contributors to the response, at least in some compositions. However, only the dimensional stacking technique enabled a direct and quantitative comparison of the evolution of the different contributors across the phase diagram. Specifically, a poling behavior-signature of the presence of a relaxor component-was observed in all compositions. In addition, the existence of a domain glass state was also identified in all the compositions containing the ferroelectric end member.

The dimensional stacking technique is valid for the correlated analysis of any multidimensional dataset, in a wide range of fields. It should prove of particular interest for the analysis of the functional response of all materials of complex compositions, as well as design of new functional materials.

\section{METHODS}

Local relaxation of electromechanical response of $(1-x) \mathrm{Pb}\left(\mathrm{Mg}_{1 / 3} \mathrm{Nb}_{2 / 3}\right) \mathrm{O}_{3}$ $-x \mathrm{PbTiO}_{3}$ single crystals has been studied by BE-PFM, following the procedure previously reported in ref. ${ }^{43}$ The BE-PFM was performed on an Asylum Research Cypher S, in ambient conditions. The tips used were metalcoated Budget Sensors $(k \approx 1 \mathrm{~N} / \mathrm{m})$ with a free in-air resonance of $\approx 75 \mathrm{kHz}$. The measurements were performed over a $50 \times 50$ points grid, covering a $2 \mu \mathrm{m} \times 2 \mu \mathrm{m}$ area. At each point, a BE-PFM waveform was overlaid with a voltage, and applied to the tip. The evolution of the piezoresponse was probed both as a function of time up to $0.9 \mathrm{~s}$ in time steps of $0.025 \mathrm{~s}$, and perturbation voltage. The specific time, voltage and compositiondependent response $D^{p_{v}, p_{c}}(t, z)$ was measured out-of-field, after the application of each of the ten alternating increasing positive and negative voltages, equal to and above the coercive voltage.

The measurements were repeated for the three compositions studied in this work, 001-cut PMN, PMN-0.36PT, and PMN-0.40PT. The single crystals of PMN-xPT have been grown by the Bridgman method, and the specific compositions were chosen to represent the phase diagram of PMN-XPT: a pure relaxor, a composition near the MPB, and lastly with a large ferroelectric content.

Dimensional stacking and Big Data analytics were implemented in a Jupyter notebook, using the Python programming language. The standard open source $M L$ and dimensionality reduction libraries were used for the analysis.

\section{DATA AVAILABILITY}

The data that support the findings of this study are available from the corresponding author upon request.

\section{CODE AVAILABILITY}

The code that support the findings of this study are available from the corresponding author upon request.

\section{ACKNOWLEDGEMENTS}

N.B.G. and L.A. Griffin gratefully acknowledge support from the US National Science Foundation through grant \# DMR-1255379, from the Defense Threat Reduction Agency (DTRA) through grant \# HDTRA1-15-0035, and from the Center for the Science and Technology of Advanced Materials and Interfaces (STAMI) at the Georgia Institute of Technology. I.G. acknowledges the support of the Division II of the Swiss National Science Foundation under project 200021_178782. The piezoresponse measurements were in part performed at the Center for Nanophase Materials Sciences at Oak Ridge National Laboratory, which is a DOE Office of Science User Facility.

\section{AUTHOR CONTRIBUTIONS}

L.A.G. performed the piezoresponse experiments and performed the analysis. N.B.G. devised the experiments. S.Z. prepared the samples. N.B.G. and I.G. devised the analysis and interpreted the data. All authors contributed to the writing of the paper. L.A.G. and I.G. are equal contributors.

\section{ADDITIONAL INFORMATION}

Supplementary Information accompanies the paper on the npj Computational Materials website (https://doi.org/10.1038/s41524-019-0222-z).

Competing interests: The authors declare no competing interests.

Publisher's note: Springer Nature remains neutral with regard to jurisdictional claims in published maps and institutional affiliations.

\section{REFERENCES}

1. Zeng, W. et al. Fiber-based wearable electronics: a review of materials, fabrication, devices, and applications. Adv. Mater. 26, 5310-5336 (2014).

2. Amjadi, M., Kyung, K.-U., Park, I. \& Sitti, M. Stretchable, skin-mountable, and wearable strain sensors and their potential applications: a review. Adv. Funct. Mater. 26, 1678-1698 (2016).

3. Ramadan, K. S., Sameoto, D. \& Evoy, S. A review of piezoelectric polymers as functional materials for electromechanical transducers. Smart Mater. Struct. 23, 033001 (2014).

4. Cheng, F., Liang, J., Tao, Z. \& Chen, J. Functional materials for rechargeable batteries. Adv. Mater. 23, 1695-1715 (2011).

5. Guo, X. et al. Recent developments of aprotic lithium-oxygen batteries: functional materials determine the electrochemical performance. Sci. Bull. 62, 442-452 (2017).

6. Saparov, B. \& Mitzi, D. B. Organic-inorganic perovskites: structural versatility for functional materials design. Chem. Rev. 116, 4558-4596 (2016).

7. Zinkle, S. \& Snead, L. Designing radiation resistance in materials for fusion energy Annu. Rev. Mater. Res. 44, 241-267 (2014).

8. Goesmann, H. \& Feldmann, C. Nanoparticulate functional materials. Angew. Chem. Int. Ed. 49, 1362-1395 (2010).

9. Kim, J. et al. Miniaturized battery-free wireless systems for wearable pulse oximetry. Adv. Funct. Mater. 27, 1604373 (2017).

10. Park, S. E. \& Shrout, T. R. Ultrahigh strain and piezoelectric behavior in relaxor based ferroelectric single crystals. J. Appl. Phys. 82, 1804-1811 (1997).

11. Cheng, K. C. et al. Single crystal PMN-0.33PT/epoxy 1-3 composites for ultrasonic transducer applications. IEEE Trans. Ultrason. Ferroelectr. Freq. Control 50, 0885-3010 (2003). 
12. Ewart, L. M., Mclaughlin, E. A., Robinson, H. C., Stace, J. J. \& Amin, A. A. Mechanical and electromechanical properties of pmnt single crystals for naval sonar transducers. IEEE Trans. Ultrason. Ferroelectr. Freq. Control 54, 0885-3010 (2007).

13. Li, F. et al. Composition and phase dependence of the intrinsic and extrinsic piezoelectric activity of domain engineered $\mathrm{Pb}\left(\mathrm{Mg}_{1 / 3} \mathrm{Nb}_{2 / 3}\right) \mathrm{O}_{3}-\mathrm{xPbTiO}$ crystals. J. Appl. Phys. 108, 034106 (2010).

14. Li, F., Zhang, S., Damjanovic, D., Chen, L.-Q. \& Shrout, T. Local structural heterogeneity and electromechanical responses of ferroelectrics: Learning from relaxor ferroelectrics. Adv. Funct. Mater. 0, 1801504 (2018).

15. Finkel, P., Benjamin, K. \& Amin, A. Large strain transduction utilizing phase transition in relaxor-ferroelectric $\mathrm{Pb}\left(\mathrm{In}_{1 / 2} \mathrm{Nb}_{1 / 2}\right) \mathrm{O}_{3}-\mathrm{Pb}\left(\mathrm{Mg}_{1 / 3} \mathrm{Nb}_{2 / 3}\right) \mathrm{O}_{3}-\mathrm{PbTiO}_{3}$ single crystals. Appl. Phys. Lett. 98, 192902 (2011).

16. Kalinin, S. V. et al. Direct evidence of mesoscopic dynamic heterogeneities at the surfaces of ergodic ferroelectric relaxors. Phys. Rev. B 81, 064107 (2010).

17. Manley, M. E. et al. Phonon localization drives polar nanoregions in a relaxor ferroelectric. Nat. Commun. 5, 3683 (2014).

18. Jiang, F., Kojima, S., Zhao, C. \& Feng, C. Chemical ordering in lanthanum-doped lead magnesium niobate relaxor ferroelectrics probed by A1g raman mode. Appl. Phys. Lett. 79, 3938-3940 (2001).

19. Chen, Y. H., Hirose, S., Viehland, D., Takahashi, S. \& Uchino, K. Mn-modified Pb $\left(\mathrm{Mg}_{1 / 3} \mathrm{Nb}_{2 / 3}\right) \mathrm{O}_{3}-\mathrm{xPTiO}_{3}$ ceramics: improved mechanical quality factors for highpower transducer applications. Jpn. J. Appl. Phys. 39, 4843 (2000).

20. Feng, Z., Tan, O. K., Zhu, W., Jia, Y. \& Luo, H. Aging-induced giant recoverable electrostrain in fe-doped $0.62 \mathrm{~Pb}\left(\mathrm{Mg}_{1 / 3} \mathrm{Nb}_{2 / 3}\right) \mathrm{O}_{3}-\mathrm{x} 0.38 \mathrm{PbTiO}_{3}$ single crystals. Appl. Phys. Lett. 92, 142910 (2008).

21. Zhang, S., Luo, J., Hackenberger, W. \& Shrout, T. R. Characterization of $\mathrm{Pb}\left(\ln _{1 / 2} \mathrm{Nb}_{1 /}\right.$ 2) $\mathrm{O}_{3}-\mathrm{Pb}\left(\mathrm{Mg}_{1 / 3} \mathrm{Nb}_{2 / 3}\right) \mathrm{O}_{3}-\mathrm{PbTiO}_{3}$ ferroelectric crystal with enhanced phase transition temperatures. J. Appl. Phys. 104, 064106 (2008).

22. Li, F. et al. Ultrahigh piezoelectricity in ferroelectric ceramics by design. Nat. Mater. 17, 349-354 (2018).

23. Chen, Z. et al. Stress-induced reversible and irreversible ferroelectric domain switching. Appl. Phys. Lett. 112, 152901 (2018).

24. Chen, Z. et al. Kinetics of domain switching by mechanical and electrical stimulation in relaxor-based ferroelectrics. Phys. Rev. Appl. 8, 064005 (2017).

25. Chen, Z. et al. Facilitation of ferroelectric switching via mechanical manipulation of hierarchical nanoscale domain structures. Phys. Rev. Lett. 118, 017601 (2017).

26. Kling, J. et al. In situ transmission electron microscopy of electric field-triggered reversible domain formation in Bi-based lead-free piezoceramics. J. Am. Ceram. Soc. 93, 2452-2455 (2010)

27. Ma, C. \& Tan, X. In situ transmission electron microscopy study on the phase transitions in lead-free (1-x)( $\left.\mathrm{Bi}_{1 / 2} \mathrm{Na}_{1 / 2}\right) \mathrm{TiO}_{3}-\mathrm{BaTiO}_{3}$ ceramics. J. Am. Ceram. Soc. 94, 4040-4044 (2011).

28. Shvartsman, V., Dkhil, B. \& Kholkin, A. Mesoscale domains and nature of the relaxor state by piezoresponse force microscopy. Annu. Rev. Mater. Res. 43, 423-449 (2013).

29. Simagina, L. V., Volk, T. R., Bodnarchuk, Y. V., Gainutdinov, R. V. \& Ivleva, L. I. Backswitching effect in relaxor SBN crystals, studied by PFM-spectroscopy. Ferroelectr. Lett. Sect. 44, 65-72 (2017).

30. Garten, L. M. et al. Relaxor ferroelectric behavior in barium strontium titanate. J. Am. Ceram. Soc. 99, 1645-1650 (2016).

31. Wang, Y., Yuan, G., Luo, H., Li, J. \& Viehland, D. Phase transition in the near-surface region of ternary $\mathrm{Pb}\left(\mathrm{In}_{1 / 2} \mathrm{Nb}_{1 / 2}\right) \mathrm{O}_{3}-\mathrm{Pb}\left(\mathrm{Mg}_{1 / 3} \mathrm{Nb}_{2 / 3}\right) \mathrm{O}_{3}-\mathrm{PbTiO}_{3}$ relaxor ferroelectric crystals. Phys. Rev. Appl. 8, 034032 (2017)

32. Dittmer, R. et al. Ergodicity reflected in macroscopic and microscopic fielddependent behavior of BNT-based relaxors. J. Appl. Phys. 115, 084111 (2014).

33. Li, Q. et al. Piezoresponse force microscopy studies on the domain structures and local switching behavior of $\mathrm{Pb}\left(\mathrm{In}_{1 / 2} \mathrm{Nb}_{1 / 2}\right) \mathrm{O}_{3}-\mathrm{Pb}\left(\mathrm{Mg}_{1 / 3} \mathrm{Nb}_{2 / 3}\right) \mathrm{O}_{3}-\mathrm{PbTiO}_{3}$ single crystals. J. Appl. Phys. 112, 052006 (2012).

34. Rodriguez, B. J. et al. Real space mapping of polarization dynamics and hysteresis loop formation in relaxor-ferroelectric $\mathrm{Pb}\left(\mathrm{Mg}_{1 / 3} \mathrm{Nb}_{2 / 3}\right) \mathrm{O}_{3}-\mathrm{PbTiO}_{3}$ solid solutions. J. Appl. Phys. 108, 042006 (2010).
35. Shvartsman, V. V. \& Kholkin, A. L. Evolution of nanodomains in $0.9 \mathrm{~Pb}\left(\mathrm{Mg}_{1 / 3} \mathrm{Nb}_{2 / 3}\right)$ $\mathrm{O}_{3}-0.1 \mathrm{PbTiO}_{3}$ single crystals. J. Appl. Phys. 101, 064108 (2007).

36. Kholkin, A. et al. Surface domain structures and mesoscopic phase transition in relaxor ferroelectrics. Adv. Funct. Mater. 21, 1977-1987 (2011).

37. Butler, K. T., Davies, D. W., Cartwright, H., Isayev, O. \& Walsh, A. Machine learning for molecular and materials science. Nature 559, 547-555 (2018).

38. Strelcov, E., Belianinov, A., Hsieh, Y.-H., Chu, Y.-H. \& Kalinin, S. V. Constraining data mining with physical models: voltage- and oxygen pressure-dependent transport in multiferroic nanostructures. Nano Lett. 15, 6650-6657 (2015).

39. Jesse, S. et al. Big data analytics for scanning transmission electron microscopy ptychography. Sci. Rep. 6, 26348 (2018).

40. Belianinov, A. et al. Research update: spatially resolved mapping of electronic structure on atomic level by multivariate statistical analysis. APL Mater. 2, 120701 (2014).

41. Strelcov, E. et al. Deep data analysis of conductive phenomena on complex oxide interfaces: physics from data mining. ACS Nano 8, 6449 (2014).

42. Vasudevan, R. K. et al. Mesoscopic harmonic mapping of electromechanical response in a relaxor ferroelectric. Appl. Phys. Lett. 106, 222901 (2015).

43. Vasudevan, R. K. et al. Multidimensional dynamic piezoresponse measurements: unraveling local relaxation behavior in relaxor-ferroelectrics via big data. J. Appl. Phys. 118, 072003 (2015).

44. Nataf, G. F., Barrett, N., Kreisel, J. \& Guennou, M. Raman signatures of ferroic domain walls captured by principal component analysis. J. Phys. 30, 035902 (2018).

45. Li, L. et al. Machine learning-enabled identification of material phase transitions based on experimental data: exploring collective dynamics in ferroelectric relaxors. Sci. Adv. 4, eaap8672 (2018).

46. Belianinov, A. et al. Big data and deep data in scanning and electron microscopies: deriving functionality from multidimensional data sets. Adv. Struct. Chem. Imaging 1, 6 (2015).

47. Trzmiel, J., Weron, K., Janczura, J. \& Placzek-Popko, E. Properties of the relaxation time distribution underlying the Kohlrausch-Williams-Watts photoionization of the $\mathrm{dx}$ centers in $\mathrm{Cd}_{1-\mathrm{x}} \mathrm{Mn}_{\mathrm{x}}$ Te mixed crystals. J. Phys. 21, 345801 (2009).

48. Garcia-Barriocanal, J. et al. Tailoring disorder and dimensionality: strategies for improved solid oxide fuel cell electrolytes. ChemPhysChem 10, 1003-1011 (2009).

49. Salje, E. K. H., Ding, X., Zhao, Z., Lookman, T. \& Saxena, A. Thermally activated avalanches: jamming and the progression of needle domains. Phys. Rev. B 83, 104109 (2011).

50. Salje, E. K. H., Ding, X. \& Aktas, O. Domain glass. Phys. Status Solidi B 251, 2061-2066 (2014)

51. Bokov, A. \& Ye, Z. Recent progress in relaxor ferroelectrics with perovskite structure. J. Mater. Sci. 41, 31-52 (2006).

52. Fan, Y., Iwashita, T. \& Egami, T. Energy landscape-driven non-equilibrium evolution of inherent structure in disordered material. Nat. Commun. 8, 15417 (2017).

53. Samara, G. The relaxational properties of compositionally disordered $A B O 3$ perovskites. J. Phys. 15, R367 (2003).

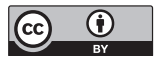

Open Access This article is licensed under a Creative Commons Attribution 4.0 International License, which permits use, sharing, adaptation, distribution and reproduction in any medium or format, as long as you give appropriate credit to the original author(s) and the source, provide a link to the Creative Commons license, and indicate if changes were made. The images or other third party material in this article are included in the article's Creative Commons license, unless indicated otherwise in a credit line to the material. If material is not included in the article's Creative Commons license and your intended use is not permitted by statutory regulation or exceeds the permitted use, you will need to obtain permission directly from the copyright holder. To view a copy of this license, visit http://creativecommons. org/licenses/by/4.0/.

(c) The Author(s) 2019 